\title{
Domination in Operations on Intuitionistic Fuzzy Graphs
}

\author{
J. John Stephan \\ Assistant professor, \\ Department of Mathematics \\ Dhanalakshmi srinivasan \\ Engineering college, \\ Perambalur
}

\author{
A. Muthaiyan \\ Assistant professor \\ P.G\& Research department of \\ Mathematics, \\ Government Arts College. \\ Ariyalur
}

\author{
N. Vinoth Kumar \\ Assistant professor, \\ Department of Mathematics, \\ M.A.M. School of Engineering, \\ Trichy
}

\begin{abstract}
In this paper we discuss several operations on intuitionistic fuzzy graph such as union, join, composition, Cartesian product and study their domination parameters.
\end{abstract}

\section{Keywords}

Intuitionistic fuzzy graph , domination, domination number

\section{INTRODUCTION}

The first definition of fuzzy graphs was proposed by Kafmann, from the fuzzy relations introduced by Zadeh. Although Rosenfeld introduced another elaborated definition, including fuzzy vertex and fuzzy edges, and several fuzzy analogs of graph theoretic concepts such as paths, cycles, connectedness and etc. The concept of domination in fuzzy graphs was investigated by A. Somasundaram, S. Somasundaram [6] and A. Somasundaram present the concepts of independent domination, total domination, connected domination of fuzzy graphs . C. Natarajan and S.K. Ayyaswamy introduce the strong (weak) domination in fuzzy graph [2]. The first definition of intuitionistic fuzzy graphs was proposed by Atanassov [1]. The concept of domination in intuitionistic fuzzy graphs was investigated by R.parvathi and G.Thamizhendhi [8]. In this paper develop the concept of Domination in operations inuitionistic fuzzy graph.

\section{DEFINITIONS}

An intuitionistic fuzzy graph (IFG) is of the form $\mathrm{G}=(\mathrm{V}, \mathrm{E})$, where $\mathrm{V}=\left\{\mathrm{v}_{1}, \mathrm{v}_{2}, \ldots, \mathrm{v}_{\mathrm{n}}\right\}$ such that $\mu_{1}: V \rightarrow[0,1], \gamma_{1}: V \rightarrow\{0,1]$ denote the degree of membership and nonmember ship of the element $v_{i} \in V$ respectively and $0 \leq \mu_{1}+\gamma_{1} \leq 1$ for every $v_{i} \in V,(\mathrm{i}=1,2, \ldots \mathrm{n}) \quad$ (ii) $\mathrm{E} \subseteq \mathrm{V} \times \mathrm{V} \quad$ where $\mu_{2}: V \times V \rightarrow[0,1]$ and $\gamma_{2}: V \times V \rightarrow[0,1]$ are such

$$
\mu_{2}\left(v_{i} v_{j}\right) \leq \mu_{1}\left(v_{1}\right) \wedge \mu_{1}\left(v_{j}\right)
$$

that $\gamma_{2}\left(v_{i} v_{j}\right) \leq \gamma_{1}\left(v_{1}\right) \vee \gamma_{1}\left(v_{j}\right)$, and

$$
0 \leq \mu_{2}\left(v_{i} v_{j}\right)+\gamma_{2}\left(v_{i} v_{j}\right) \leq 1 \text {. }
$$

$$
\begin{array}{lrrrrr}
\text { An } \quad \text { arc }\left(v_{i}, v_{j}\right) & \text { of } & \text { an } & \text { IFG } & \text { G } & \text { is } \\
\text { called } \quad \text { an } \quad \text { strong } & & \text { arc } & & \text { if } \\
\mu_{2}\left(v_{i}, v_{j}\right)=\mu_{1}\left(v_{i}\right) \wedge \mu_{1}\left(v_{j}\right), & & & & \\
\gamma_{2}\left(v_{i}, v_{j}\right)=\gamma_{1}\left(v_{i}\right) \vee \gamma_{1}\left(v_{j}\right) . & & &
\end{array}
$$

Let $\mathrm{G}=(\mathrm{V}, \mathrm{E})$ be an IFG on $\mathrm{V}$. Let $u, v \in V$ we say that $u$ dominates $v$ in $G$ if

$$
\begin{aligned}
& \mu_{2}\left(v_{i} v_{j}\right)=\mu_{1}\left(v_{1}\right) \wedge \mu_{1}\left(v_{j}\right), \\
& \gamma_{2}\left(v_{i} v_{j}\right)=\gamma_{1}\left(v_{1}\right) \vee \gamma_{1}\left(v_{j}\right)
\end{aligned}
$$

A sub set $\mathrm{S}$ of $\mathrm{V}$ is called a dominating set in G if for every $v \in V-S$, there exist $u \in S$ such that $\mathrm{u}$ dominates $\mathrm{v}$.

The minimum cardinality of a dominating set in $G$ is called the domination number of $G$ and is denoted by $\gamma(G)$ or $\gamma$.

A vertex $u$ of an IFG is said to be an isolated vertex if $\mu_{2}\left(v_{i} v_{j}\right)<\mu_{1}\left(v_{1}\right) \wedge \mu_{1}\left(v_{j}\right), \quad$ for all $v \in V-\{u\}$.

Let $G=(V, E)$ be an IFG on V. A subset $\mathrm{S}$ of $\mathrm{V}$ is said to be an independent set if $\mu_{2}\left(v_{i} v_{j}\right)<\mu_{1}\left(v_{1}\right) \wedge \mu_{1}\left(v_{j}\right)$, for all $u, v \in S . S$ is said $\gamma_{2}\left(v_{i} v_{j}\right)<\gamma_{1}\left(v_{1}\right) \vee \gamma_{1}\left(v_{j}\right)$

to be a maximal independent set if $S \cup\{v\}$ is not an independent set for any $v \in V-S$.

The maximum cardinality of an independent set in $G$ is called the independence number of $G$ is denoted by $\beta_{0}(G)$.the maximum fuzzy cardinality of an independent dominating ser of $\mathrm{G}$ is called the independent dominating number of $\mathrm{G}$ and is denoted by $\gamma_{i}(G)$.

\section{MAIN RESULT}

\subsection{Union of IFG}

Let $\mathrm{G}_{1}=\left(\mathrm{V}_{1}, \mathrm{E}_{1}\right)$ and $\mathrm{G}_{2}=\left(\mathrm{V}_{2}, \mathrm{E}_{2}\right)$ be intuitionistic fuzzy graphs on $\mathrm{V}_{1}, \mathrm{~V}_{2}$ respectively with $V_{1} \cap V_{2}=\phi$. The union of $\mathrm{G}_{1}$ and $\mathrm{G}_{2}$ denoted by $G_{1}+G_{2}$, is the intuitionistic fuzzy graph $\mathrm{G}$ on $V_{1} \cup V_{2}$ defined by $G=\left(G_{1} \cup G_{2}\right)=\left(\left(\mu_{1} \cup \mu_{1}^{\prime}\right),\left(\gamma_{1} \cup \gamma_{1}^{\prime}\right),\left(\mu_{2} \cup \mu_{2}^{\prime}\right),\left(\gamma_{2} \cup \gamma_{2}^{\prime}\right)\right)$ where

$$
\left(\mu_{1} \cup \mu_{1}^{\prime}\right)(u)=\left\{\begin{array}{l}
\mu_{1}(u) \text { if } u \in V_{1} \\
\mu_{1}^{\prime}(u) \text { if } u \in V_{2}
\end{array}\right.
$$




$$
\begin{aligned}
& \left(\gamma_{1} \cup \gamma_{1}^{\prime}\right)(u)=\left\{\begin{array}{l}
\gamma_{1}(u) \text { if } u \in V_{1} \\
\gamma_{1}^{\prime}(u) \text { if } u \in V_{2}
\end{array}\right. \\
& \left(\mu_{2} \cup \mu_{2}^{\prime}\right)(u v)=\left\{\begin{array}{l}
\mu_{2}(u v) \text { if } u v \in E_{1} \\
\mu_{2}^{\prime}(u v) \text { if } u v \in E_{2} \\
0 \text { otherwise }
\end{array}\right. \text { and } \\
& \left(\gamma_{2} \cup \gamma_{2}^{\prime}\right)(u v)=\left\{\begin{array}{l}
\gamma_{2}(u v) \text { if } u v \in E_{1} \\
\gamma_{2}^{\prime}(u v) \text { if } u v \in E_{2} \\
0 \text { otherwise }
\end{array}\right.
\end{aligned}
$$

Remark: since dominating set D of $G_{1} \cup G_{2}$ is of the form $D=D_{1} \cup D_{2}$, where $\mathrm{D}_{1}$ is the dominating set of $\mathrm{G}_{1}$ and $\mathrm{D}_{2}$ is the dominating set of $\mathrm{G}_{2}$, it follows that $\gamma\left(G_{1} \cup G_{2}\right)=\gamma\left(G_{1}\right)+\gamma\left(G_{2}\right)$.

Example:
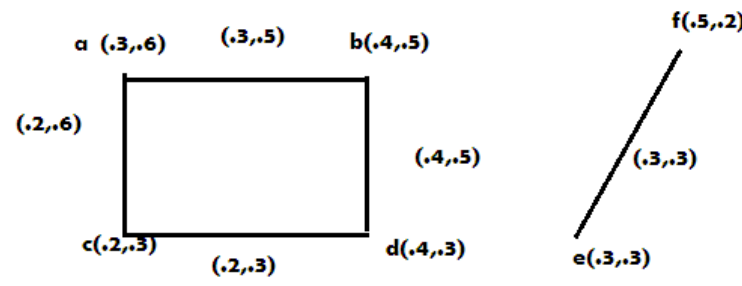

G

$\mathbf{G}_{2}$
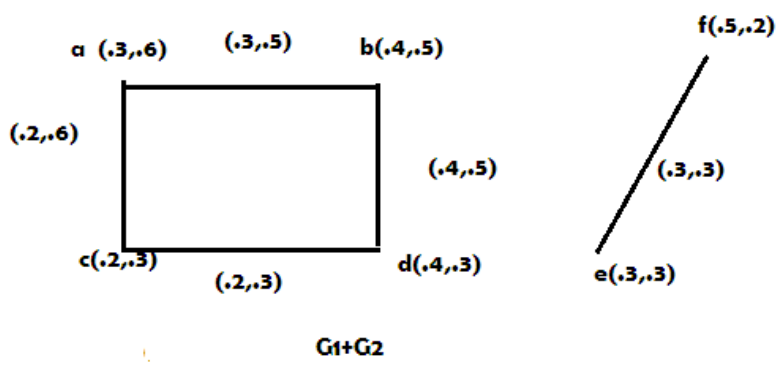

In Fig. the dominating set of $\mathrm{G}_{1}=\{\mathrm{a}, \mathrm{d}\}$ and $\gamma\left(G_{1}\right)=(.7, .9)$ $\gamma\left(G_{2}\right)=(.3, .3)$

In Fig. the dominating set of $\mathrm{G}_{2}=\{\mathrm{e}\}$ and The dominating set of $\mathrm{G}_{1}+\mathrm{G}_{2}$ is $\{\mathrm{a}, \mathrm{d}, \mathrm{e}\}$ and $\gamma\left(G_{2}+G_{2}\right)=(.7, .9)$

\subsection{Join of IFG}

Let $\mathrm{G}_{1}=\left(\mathrm{V}_{1}, \mathrm{E}_{1}\right)$ and $\mathrm{G}_{2}=\left(\mathrm{V}_{2}, \mathrm{E}_{2}\right)$ be intuitionistic fuzzy graphs on $\mathrm{V}_{1}, \mathrm{~V}_{2}$ respectively with $V_{1} \cap V_{2}=\phi$. The join of $\mathrm{G}_{1}$ and $\mathrm{G}_{2}$ is the intuitionistic fuzzy graph $\mathrm{G}$ on $V_{1} \cup V_{2}$ defined by $G=\left(G_{1}+G_{2}\right)=\left(\left(\mu_{1}+\mu_{1}^{\prime}\right),\left(\gamma_{1}+\gamma_{1}^{\prime}\right),\left(\mu_{2}+\mu_{2}^{\prime}\right),\left(\gamma_{2}+\gamma_{2}^{\prime}\right)\right)$ where

$$
\begin{aligned}
& \left(\mu_{1}+\mu_{1}^{\prime}\right)(u)=\left\{\begin{array}{l}
\mu_{1}(u) \text { if } u \in V_{1} \\
\mu_{1}^{\prime}(u) \text { if } u \in V_{2}
\end{array}\right. \\
& \left(\gamma_{1}+\gamma_{1}^{\prime}\right)(u)=\left\{\begin{array}{l}
\gamma_{1}(u) \text { if } u \in V_{1} \\
\gamma_{1}^{\prime}(u) \text { if } u \in V_{2}
\end{array}\right.
\end{aligned}
$$$$
\left(\mu_{2}+\mu_{2}^{\prime}\right)(u v)=\left\{\begin{array}{l}
\mu_{2}(u v) \text { if } u v \in E_{1} \\
\mu_{2}^{\prime}(u v) \text { if } u v \in E_{2} \\
\mu_{1}(u) \wedge \mu_{1}^{\prime}(v) \text { if } u \in V_{1} \& v \in V_{2}
\end{array}\right.
$$

and

$$
\left(\gamma_{2}+\gamma_{2}^{\prime}\right)(u v)=\left\{\begin{array}{l}
\gamma_{2}(u v) \text { if } u v \in E_{1} \\
\gamma_{2}^{\prime}(u v) \text { if } u v \in E_{2} \\
\gamma_{1}(u) \vee \gamma_{1}^{\prime}(v) \text { if } u \in V_{1} \& v \in V
\end{array}\right.
$$

\subsubsection{Theorem}

Let $G_{1}=\left(V_{1}, E_{1}\right)$ and $G_{2}=\left(V_{2}, E_{2}\right)$ be two IFG on $\mathrm{V}_{1}$ and $\mathrm{V}_{2}$ respectively with $V_{1} \cap V_{2}=\phi$ then

$$
\begin{array}{ll}
\text { i. } & \gamma\left(G_{1}+G_{2}\right)=\min \left\{\gamma\left(G_{1}\right), \gamma\left(G_{2}\right),\left\{\mu_{1}(u)+\mu_{1}^{\prime}(v), \gamma_{1}(u)+\gamma_{1}^{\prime}(v)\right\}\right\} \\
& \text { where } u_{1} \in V_{1}, v \in V_{2} \\
\text { ii. } & \gamma_{i}\left(G_{1}+G_{2}\right)=\min \left\{\gamma_{1}\left(G_{1}\right), \gamma_{1}\left(G_{2}\right)\right\}
\end{array}
$$

Proof

(i). It follows from the definition of $G_{1}+G_{2}$ any edges of the form uv, where $u_{1} \in V_{1}, v \in V_{2}$ is an effective edge. Hence any vertex of $V_{1}$ dominates all the vertices of $\mathrm{V}_{2}$. Now let $\mathrm{D}$ be any minimal dominating set of $G_{1}+G_{2}$. Then D is of the following form

1) $\mathrm{D}=\mathrm{D}_{1}$ where $\mathrm{D}_{1}$ is a minimal dominating set of $\mathrm{G}_{1}$

2) $\mathrm{D}=\mathrm{D}_{2}$ where $\mathrm{D}_{2}$ is a minimal dominating set of $\mathrm{G}_{2}$

3) $\mathrm{D}=\{\mathrm{u}, \mathrm{v}\}$ where $u \in V_{1}$ and $v \in V_{2},\{\mathrm{u}\}$ is not a dominating set of $G_{1}$ and $\{u\}$ is not a dominating set of $\mathrm{G}_{2}$.

Hence

$\gamma\left(G_{1}+G_{2}\right)=\min \left\{\gamma\left(G_{1}\right), \gamma\left(G_{2}\right),\left\{\mu_{1}(u)+\mu_{1}^{\prime}(v), \gamma_{1}(u)+\gamma_{1}^{\prime}(v)\right\}\right\}$ where $u_{1} \in V_{1}, v \in V_{2}$.

(ii). Since every vertex of $\mathrm{V}_{1}$ dominates every vertex of $\mathrm{V}_{2}$ in $\mathrm{G}_{1}+\mathrm{G}_{2}$. Any independent set in $G_{1}+G_{2}$ is either a subset of $\mathrm{V}_{1}$ or a subset of $\mathrm{V}_{2}$. Hence any minimal dominating set $\mathrm{D}$ of $G_{1}+G_{2}$ is one of the following forms

1) $\mathrm{D}=\mathrm{D}_{1}$, where $\mathrm{D}_{1}$ is a minimal independent dominating set of $\mathrm{G}_{1}$

2) $\mathrm{D}=\mathrm{D}_{2}$, where $\mathrm{D}_{2}$ is a minimal independent dominating set of $\mathrm{G}_{2}$.

Thus $\gamma_{i}\left(G_{1}+G_{2}\right)=\min \left\{\gamma_{1}\left(G_{1}\right), \gamma_{1}\left(G_{2}\right)\right\}$

\section{Example:}




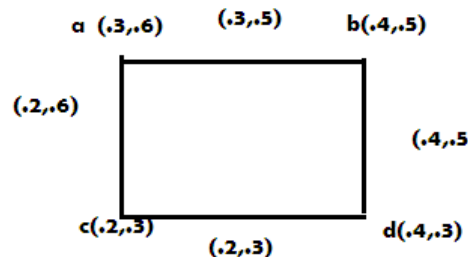

G1

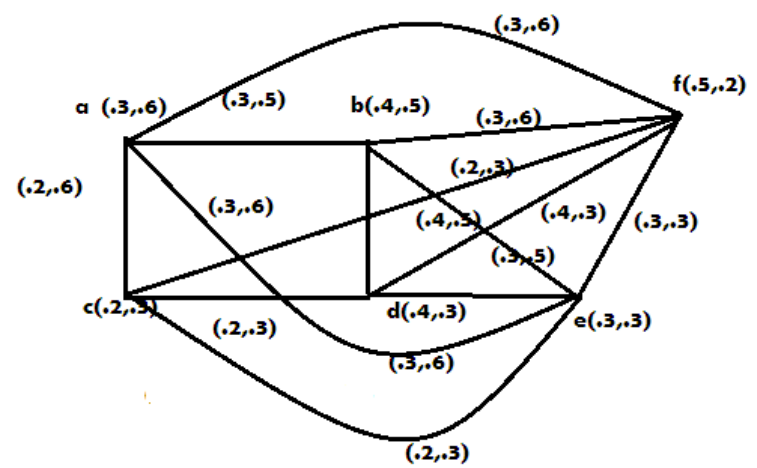

$\mathrm{G}_{1}+\mathrm{G}_{2}$

The dominating set is $\{\mathrm{e}\}$ and $\gamma\left(G_{1}+G_{2}\right)=(.3, .3)$

\subsection{Composition of IFG}

Let $\mathrm{G}_{1}=\left(\mathrm{V}_{1}, \mathrm{E}_{1}\right)$ and $\mathrm{G}_{2}=\left(\mathrm{V}_{2}, \mathrm{E}_{2}\right)$ be intuitionistic fuzzy graphs on $V_{1}, V_{2}$ respectively then the composition of $G_{1}$ and $G_{2}$ denoted by $G_{1} \circ G_{2}$, is the intuitionistic fuzzy graph $\mathrm{G}$ on $V_{1} \times V_{2} \quad$ defined by $G=\left(G_{1} \circ G_{2}\right)=\left(\left(\mu_{1} \circ \mu_{1}^{\prime}\right),\left(\gamma_{1} \circ \gamma_{1}^{\prime}\right),\left(\mu_{2} \circ \mu_{2}^{\prime}\right),\left(\gamma_{2} \circ \gamma_{2}^{\prime}\right)\right)$ wh ere

$\left(\mu_{1} \circ \mu_{1}^{\prime}\right)\left(u_{1}, u_{2}\right)=\mu_{1}\left(u_{1}\right) \wedge \mu_{1}^{\prime}\left(u_{2}\right)$
$\left(\gamma_{1} \circ \gamma_{1}^{\prime}\right)\left(u_{1}, u_{2}\right)=\gamma_{1}\left(u_{1}\right) \vee \gamma_{1}^{\prime}\left(u_{2}\right)$

$\left(\mu_{2} \circ \mu_{2}^{\prime}\right)\left(u_{1} u_{2}\right)\left(v_{1} v_{2}\right)=\left\{\begin{array}{l}\mu_{1}\left(u_{1}\right) \wedge \mu_{2}^{\prime}\left(u_{2} v_{2}\right) \text { if } u_{1}=v_{1} \& u_{2} \neq v_{2} \\ \mu_{1}^{\prime}\left(u_{2}\right) \wedge \mu_{1}^{\prime}\left(v_{2}\right) \wedge \mu_{2}\left(u_{1} v_{1}\right) \text { otherwise }\end{array}\right.$ and

$\left(\gamma_{2} \cup \gamma_{2}^{\prime}\right)\left(u_{1} u_{2}\right)\left(v_{1} v_{2}\right)=\left\{\begin{array}{l}\gamma_{1}\left(u_{1}\right) \vee \gamma_{2}^{\prime}\left(u_{2} v_{2}\right) \text { if } u_{1}=v_{1} \& u_{2} \neq v_{2} \\ \gamma_{1}^{\prime}\left(u_{2}\right) \vee \gamma_{1}^{\prime}\left(v_{2}\right) \vee \gamma_{2}\left(u_{1} v_{1}\right) \text { otherwise }\end{array}\right.$

\subsubsection{Theorem}

Let $\mathrm{D}_{1}$ and $\mathrm{D}_{2}$ be dominating sets of the intuitionistic fuzzy graph $G_{1}=\left(V_{1}, E_{1}\right)$ and $G_{2}\left(V_{2}, E_{2}\right)$ respectively. Then $D_{1} \times D_{2}$ is a dominating set of $G_{1} \circ G_{2}$.

Proof:

$$
\text { Let }(u, v) \in D_{1} \times D_{2} \text {. Then } u \in D_{1} \text { or } v \notin D_{2}
$$

Case (i) $u \notin D_{1} a n d v \in D_{2}$

Let $u_{1} \in D_{1}$ be such that $\mathrm{u}_{1}$ dominates v. Then

$$
\begin{aligned}
& \mu_{2}\left(u u_{1}\right)=\mu_{1}(u) \wedge \mu_{1}\left(u_{1}\right) \text { and } \\
& \gamma_{2}\left(u u_{1}\right)=\gamma_{1}(u) \wedge \gamma_{1}\left(u_{1}\right)
\end{aligned}
$$

Now $\left(u_{1}, v\right) \in D_{1} \times D_{2}$ and

$$
\begin{aligned}
\left(\mu_{2} \circ \mu_{2}^{\prime}\right)((u, v) & \left.\left(u_{1}, v\right)\right)=\mu_{2}\left(u u_{1}\right) \wedge \mu_{1}^{\prime}(v) \\
= & \mu_{1}(u) \wedge \mu_{1}\left(u_{1}\right) \wedge \mu_{1}^{\prime}(v) \\
= & \mu_{1}(u) \wedge \mu_{1}^{\prime}(v) \wedge \mu_{1}\left(u_{1}\right) \wedge \mu_{1}^{\prime}(v) \\
& =\left(\mu_{1} \circ \mu_{1}^{\prime}\right)(u, v) \wedge\left(\mu_{1} \circ \mu_{1}^{\prime}\right)\left(u_{1}, v_{1}\right)
\end{aligned}
$$

And

$$
\begin{aligned}
\left(\gamma_{2} \circ \gamma_{2}^{\prime}\right)((u, v) & \left.\left(u_{1}, v\right)\right)=\gamma_{2}\left(u u_{1}\right) \vee \gamma_{1}^{\prime}(v) \\
& =\gamma_{1}(u) \vee \gamma_{1}\left(u_{1}\right) \vee \mu_{1}^{\prime}(v) \\
& =\gamma_{1}(u) \vee \gamma_{1}^{\prime}(v) \vee \gamma_{1}\left(u_{1}\right) \vee \gamma_{1}^{\prime}(v) \\
& =\left(\gamma_{1} \circ \gamma_{1}^{\prime}\right)(u, v) \vee\left(\gamma_{1} \circ \gamma_{1}^{\prime}\right)\left(u_{1}, v_{1}\right)
\end{aligned}
$$

Hence $\left(u_{1}, v\right)$ dominates (u,v) in $G_{1} \circ G_{2}$

Case (ii) $u \in D_{1}$ and $v \notin D_{2}$

Let $v_{1} \in D_{2}$ be such that $\mathrm{v}_{1}$ dominates $\mathrm{v}$. Then

$$
\begin{aligned}
& \mu_{2}^{\prime}\left(v_{1} v\right)=\mu_{1}^{\prime}\left(v_{1}\right) \wedge \mu_{1}^{\prime}(v) \text { and } \\
& \gamma_{2}^{\prime}\left(v_{1} v\right)=\gamma_{1}^{\prime}\left(v_{1}\right) \vee \gamma_{1}^{\prime}(v)
\end{aligned}
$$

Now $\left(u, v_{1}\right) \in D_{1} \times D_{2}$ and

$$
\begin{aligned}
\left(\mu_{2} \circ \mu_{2}^{\prime}\right)\left((u, v)\left(u, v_{1}\right)\right)=\mu_{1}(u) \wedge \mu_{2}^{\prime}\left(u v_{1}\right) & \\
= & \mu_{1}(u) \wedge \mu_{1}^{\prime}\left(v_{1}\right) \wedge \mu_{1}^{\prime}(v) \\
= & \mu_{1}(u) \wedge \mu_{1}^{\prime}(v) \wedge \mu_{1}(u) \wedge \mu_{1}^{\prime}\left(v_{1}\right) \\
= & \left(\mu_{1} \circ \mu_{1}^{\prime}\right)(u, v) \wedge\left(\mu_{1} \circ \mu_{1}^{\prime}\right)\left(v, v_{1}\right)
\end{aligned}
$$

And

$$
\begin{aligned}
\left(\gamma_{2} \circ \gamma_{2}^{\prime}\right)\left((u, v)\left(u, v_{1}\right)\right) & =\gamma_{1}(u) \vee \gamma_{2}^{\prime}\left(u v_{1}\right) \\
= & \gamma_{1}(u) \vee \gamma_{1}^{\prime}\left(v_{1}\right) \vee \gamma_{1}^{\prime}(v) \\
= & \gamma_{1}(u) \vee \gamma_{1}^{\prime}(v) \vee \gamma_{1}(u) \vee \gamma_{1}^{\prime}\left(v_{1}\right) \\
= & \left(\gamma_{1} \circ \gamma_{1}^{\prime}\right)(u, v) \vee\left(\gamma_{1} \circ \gamma_{1}^{\prime}\right)\left(v, v_{1}\right)
\end{aligned}
$$

Hence $\left(u, v_{1}\right)$ dominates $(\mathrm{u}, \mathrm{v})$ in $G_{1} \circ G_{2}$

Case (iii) $u \in D_{1}$ and $v \in D_{2}$

Let $u_{1} \in D_{1}$ and $v_{1} \in D_{2}$ be such that $\mathrm{u}_{1}$ dominates $\mathrm{u}$ in $\mathrm{G}_{1}$ and $\mathrm{v}_{1}$ dominates $\mathrm{v}$ in $\mathrm{G}_{2}$. Then

And

$$
\begin{aligned}
& \mu_{2}\left(u u_{1}\right)=\mu_{1}(u) \wedge \mu_{1}\left(u_{1}\right) \text { and } \\
& \gamma_{2}^{\prime}\left(u u_{1}\right)=\gamma_{1}(u) \vee \gamma_{1}\left(u_{1}\right)
\end{aligned}
$$

$$
\begin{aligned}
& \mu_{2}\left(v v_{1}\right)=\mu_{1}(v) \wedge \mu_{1}\left(v_{1}\right) \text { and } \\
& \gamma_{2}^{\prime}\left(v v_{1}\right)=\gamma_{1}(v) \vee \gamma_{1}\left(v_{1}\right)
\end{aligned}
$$

Now $\left(u_{1}, v_{1}\right) \in D_{1} \times D_{2}$ and 


$$
\begin{aligned}
\left(\mu_{2} \circ \mu_{2}^{\prime}\right)\left((u, v)\left(u_{1}, v_{1}\right)\right)= & \mu_{2}\left(u u_{1}\right) \wedge \mu_{1}^{\prime}(v) \wedge \mu_{1}^{\prime}\left(v_{1}\right) \\
& =\mu_{1}(u) \wedge \mu_{1}\left(u_{1}\right) \wedge \mu_{1}^{\prime}(v) \wedge \mu_{1}^{\prime}\left(v_{1}\right) \\
& =\mu_{1}(u) \wedge \mu_{1}^{\prime}(v) \wedge \mu_{1}\left(u_{1}\right) \wedge \mu_{1}^{\prime}\left(v_{1}\right) \\
& =\left(\mu_{1} \circ \mu_{1}^{\prime}\right)(u, v) \wedge\left(\mu_{1} \circ \mu_{1}^{\prime}\right)\left(u, v_{1}\right)
\end{aligned}
$$

And

$$
\begin{aligned}
\left(\gamma_{2} \circ \gamma_{2}^{\prime}\right)\left((u, v)\left(u_{1}, v_{1}\right)\right)= & \gamma_{2}\left(u u_{1}\right) \vee \gamma_{1}^{\prime}(v) \vee \gamma_{1}^{\prime}\left(v_{1}\right) \\
& =\gamma_{1}(u) \vee \gamma_{1}\left(u_{1}\right) \vee \gamma_{1}^{\prime}(v) \vee \gamma_{1}^{\prime}\left(v_{1}\right) \\
& =\gamma_{1}(u) \vee \gamma_{1}^{\prime}(v) \vee \gamma_{1}\left(u_{1}\right) \vee \gamma_{1}^{\prime}\left(v_{1}\right) \\
& =\left(\gamma_{1} \circ \gamma_{1}^{\prime}\right)(u, v) \vee\left(\gamma_{1} \circ \gamma_{1}^{\prime}\right)\left(u, v_{1}\right)
\end{aligned}
$$

Hence $\left(u_{1}, v_{1}\right)$ dominates $(\mathrm{u}, \quad \mathrm{v})$ in $G_{1} \circ G_{2}$. Thus $D_{1} \times D_{2}$ is a dominating set of $G_{1} \circ G_{2}$.

\section{Example:}

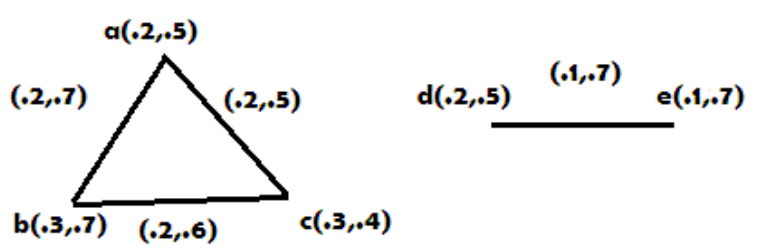

$\mathrm{G}_{1}$

$\mathrm{G}_{2}$

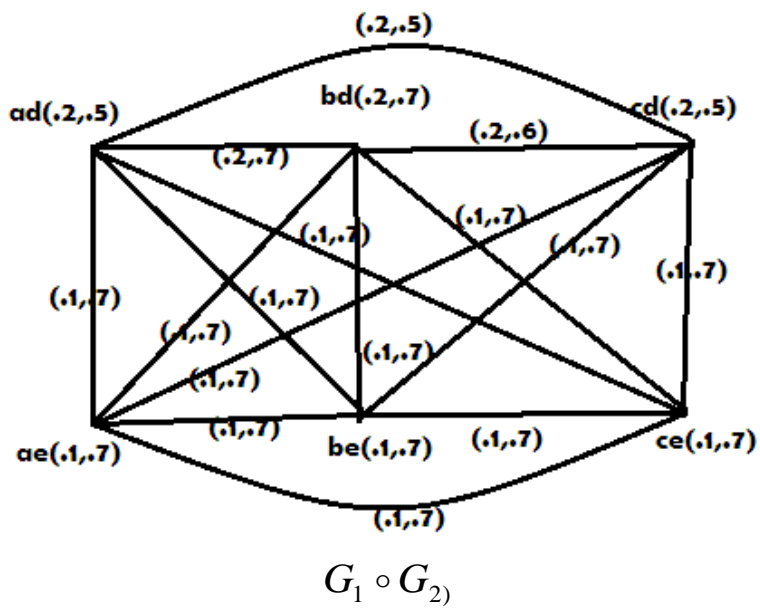

Dominating set of $G_{1} \circ G_{2)}$ is $\{\mathrm{ae}\}$

\subsection{Cartesian product of IFG}

Let $\mathrm{G}_{1}=\left(\mathrm{V}_{1}, \mathrm{E}_{1}\right)$ and $\mathrm{G}_{2}=\left(\mathrm{V}_{2}, \mathrm{E}_{2}\right)$ be intuitionistic fuzzy graphs on $\mathrm{V}_{1}, \mathrm{~V}_{2}$ respectively then the Cartesian product of $\mathrm{G}_{1}$ and $\mathrm{G}_{2}$ denoted by $G_{1} \times G_{2}$, is the intuitionistic fuzzy graph $\mathrm{G}$ on $V_{1} \times V_{2}$ defined by $G=\left(G_{1} \times G_{2}\right)=\left(\left(\mu_{1} \times \mu_{1}^{\prime}\right),\left(\gamma_{1} \times \gamma_{1}^{\prime}\right),\left(\mu_{2} \times \mu_{2}^{\prime}\right),\left(\gamma_{2} \times \gamma_{2}^{\prime}\right)\right)$ where

$$
\begin{aligned}
& \left(\mu_{1} \times \mu_{1}^{\prime}\right)\left(u_{1}, u_{2}\right)=\mu_{1}\left(u_{1}\right) \wedge \mu_{1}^{\prime}\left(u_{2}\right) \\
& \left(\gamma_{1} \times \gamma_{1}^{\prime}\right)\left(u_{1}, u_{2}\right)=\gamma_{1}\left(u_{1}\right) \vee \gamma_{1}^{\prime}\left(u_{2}\right)
\end{aligned}
$$

$\left(\mu_{2} \times \mu_{2}^{\prime}\right)\left(u_{1} u_{2}\right)\left(v_{1} v_{2}\right)=\left\{\begin{array}{l}\mu_{1}^{\prime}\left(u_{1}\right) \wedge \mu_{2}^{\prime}\left(u_{2} v_{2}\right) \text { if } u_{1}=v_{1} \\ \mu_{1}^{\prime}\left(u_{2}\right) \wedge \mu_{1}\left(u_{1} v_{1}\right) \text { if } u_{2}=v_{2} \\ \text { otherwise }\end{array}\right.$

and

$\left(\gamma_{2} \times \gamma_{2}^{\prime}\right)\left(u_{1} u_{2}\right)\left(v_{1} v_{2}\right)=\left\{\begin{array}{l}\gamma_{1}\left(u_{1}\right) \vee \gamma_{2}^{\prime}\left(u_{2} v_{2}\right) \text { if } u_{1}=v_{1} \\ \gamma_{1}^{\prime}\left(u_{2}\right) \vee \gamma_{1}\left(u_{1} v_{1}\right) \text { if } u_{2}=v_{2} \\ \text { otherwise }\end{array}\right.$

\subsubsection{Theorem}

Let $D_{1}$ and $D_{2}$ be minimum dominating set of the intuitionistic fuzzy graph $G_{1}=\left(V_{1}, E_{1}\right)$ and $G_{2}=\left(V_{2}, E_{2}\right)$ respectively. Then $\gamma_{1}\left(G_{1} \otimes G_{2}\right) \leq\left\{\left|D_{1} \times V_{2}\right|,\left|V_{1} \times D_{2}\right|\right\}$.

Proof :

We first prove that $D_{1} \times V_{2}$ is a dominating set of $G_{1} \otimes G_{2}$. Let $\left(u_{1}, u_{2}\right) \notin D_{1} \times V_{2}$. Hence $u_{1} \notin D_{1}$ since $\mathrm{D}_{1}$ is a dominating set of $\mathrm{G}_{1}$, there exist $v_{1} \in D_{1}$ such that

$$
\begin{aligned}
& \mu_{2}\left(u_{1} v_{1}\right)=\mu_{1}\left(u_{1}\right) \wedge \mu_{1}\left(v_{1}\right) \text { and } \\
& \gamma_{2}\left(u_{1} v_{1}\right)=\gamma_{1}\left(u_{1}\right) \vee \gamma_{1}\left(v_{1}\right)
\end{aligned}
$$

Now $\left(v_{1} u_{2}\right) \in\left(D_{1} \times V_{2}\right)$ and

$$
\begin{aligned}
\left(\mu_{2} \otimes \mu_{2}\right) & \left(\left(u_{1} u_{2}\right)\left(v_{1} u_{2}\right)\right)=\mu_{1}\left(u_{2}\right) \wedge \mu_{2}\left(u_{1} v_{1}\right) \\
= & \mu_{1}^{\prime}\left(u_{2}\right) \wedge \mu_{1}\left(u_{1}\right) \wedge \mu_{1}\left(v_{1}\right) \\
= & \mu_{1}\left(u_{1}\right) \wedge \mu_{1}^{\prime}\left(u_{2}\right) \wedge \mu_{1}\left(v_{1}\right) \wedge \mu_{1}^{\prime}\left(u_{2}\right) \\
= & \left(\mu_{1} \otimes \mu_{1}^{\prime}\right)\left(u_{1} u_{2}\right) \wedge\left(\mu_{1} \otimes \mu_{1}^{\prime}\right)\left(v_{1} u_{2}\right) \\
\left(\gamma_{2} \otimes \gamma_{2}^{\prime}\right) & \left(\left(u_{1} u_{2}\right)\left(v_{1} u_{2}\right)\right)=\gamma_{1}^{\prime}\left(u_{2}\right) \vee \gamma_{2}\left(u_{1} v_{1}\right) \\
= & \gamma_{1}^{\prime}\left(u_{2}\right) \vee \gamma_{1}\left(u_{1}\right) \vee \gamma_{1}\left(v_{1}\right) \\
= & \gamma_{1}\left(u_{1}\right) \vee \gamma_{1}^{\prime}\left(u_{2}\right) \vee \gamma_{1}\left(v_{1}\right) \vee \gamma_{1}^{\prime}\left(u_{2}\right) \\
= & \left(\gamma_{1} \otimes \gamma_{1}^{\prime}\right)\left(u_{1} u_{2}\right) \vee\left(\gamma_{1} \otimes \gamma_{1}^{\prime}\right)\left(v_{1} u_{2}\right)
\end{aligned}
$$

Thus $\left(\mathrm{v}_{1}, \mathrm{u}_{2}\right)$ dominates $\left(\mathrm{u}_{1}, \mathrm{u}_{2}\right)$ in $G_{1} \otimes G_{2}$. So that $D_{1} \times V_{2}$ is a dominating set of $G_{1} \otimes G_{2}$. Similarly $V_{1} \times D_{2}$ is a dominating set of $G_{1} \otimes G_{2}$ and hence it follows that $\gamma_{1}\left(G_{1} \otimes G_{2}\right) \leq\left\{\left|D_{1} \times V_{2}\right|,\left|V_{1} \times D_{2}\right|\right\}$. 


\section{Example:}
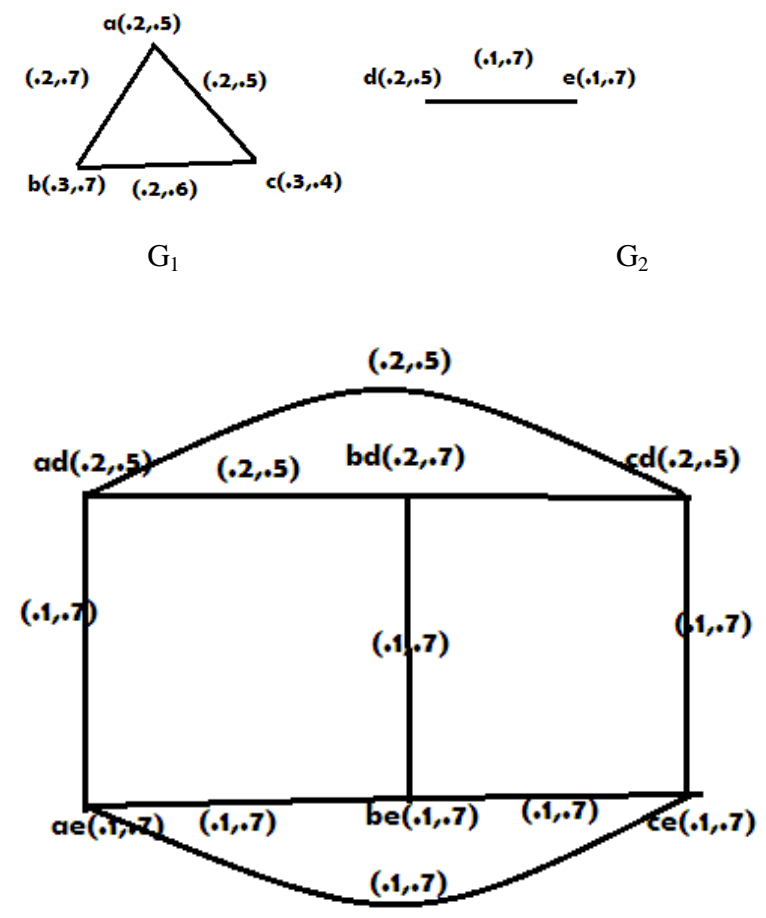

$G_{1} \times G_{2}$

Dominating set of $G_{1} \times G_{2}$ is $\{\mathbf{a e}, \mathbf{a d}\}$

\section{CONCLUSION}

In this paper we have prove some rests on operations on intuitionistic fuzzy graph . Further, the authors proposed to introduce new dominating parameters in intuitionistic fuzzy graph and apply these concepts to the intuitionistic fuzzy graph models in computer networks.

\section{REFERENCES}

[1] Atanasson , intuitionistic fuzzy set theory and applications, Physcia- verlag, New York, (199).

[2] Ayyaswamy.S, and Natarajan.C, Strong (weak) domination in fuzzy graphs, International Journal of Computational and Mathematical sciences, 2010.

[3] Balakrishnan and K.Ranganathan, A Text Book of Graph theory, Springer, 2000.

[4] Harary.F., Graph Theory, Addition Wesely, Third Printing, October 1972.

[5] Rosenfeld A. Fuzzy Graphs ,Fuzzy sets and their Applications (Acadamic Press, New York)

[6] Mordeson, J.N., and Nair, P.S., Fuzzy graphs and Fuzzy Hyper graphs, Physica-Verlag, Heidelberg, 1998, second edition, 2001.

[7] R.Parvathi and G.Thamizhendhi, Domination in Intuitionistic Fuzzy Graphs, Fourteenth Int.Conf. On IFSs, Sofia 15-16 may 2010.

[8] Somasundaram,A.,Somasundaram,S.,1998, Domination in Fuzzy Graphs-I, Pattern Recognition Letters, 19, pp. 787-791.

[9] Somasundaram, A., 2004, Domination in product Fuzzy Graph-II, Journal of Fuzzy Mathematics

[10] R.Parvathi and G.Thamizhendhi, Domination in Intuitionistic Fuzzy Graphs, Fourteenth Int.Conf. On IFSs, Sofia 15-16 may 2010 . 